\title{
A COMPARISON OF BACKCROSS AND SELFING METHODS FOR TELOCENTRIC MAPPING IN WHEAT
}

\author{
BRIAN WESTCOTT, MICHAEL D. GALE and JAMES A. McVITTIE \\ Plant Breeding Institute, Trumpington, Cambridge
}

Received 29.iv.77

\begin{abstract}
Summary
The relative efficiencies of the use of backcross and $F_{2}$ families for telocentric chromosome mapping in wheat are compared in three dominance situations. The effects of differential transmission of the telocentric and of differential zygotic viability are considered. Analysis of selfed progeny may be more complex but selfing will often be more convenient and in several genetic situations will be preferable to the more usual backcross technique.
\end{abstract}

\section{InTRODUGTION}

THE genetic linkage map in hexaploid bread wheat, Triticum aestivum $(2 n=6 x=42)$, suffers from a paucity of marker genes spread among a relatively large number of chromosomes. However wheat will tolerate aneuploidy and the availability of lines carrying telocentric chromosomes allows the location of genes relative to the centromere. Telocentric chromosomes, which lack one complete arm, are now available for both arms of all 21 wheat chromosomes and most may be maintained as pure breeding ditelocentric lines.

In order to ascertain the recombination frequency between a gene and the centromere, the progeny from a monotelodisomic plant, heterozygous at a locus carried on the telocentric, is classified both for the character controlled by the gene and for the presence of the telocentric. The telocentric may be identified either from mitotic chromosome counts of dividing root-tips or from meiotic chromosome preparations of dividing pollen mother cells.

Two types of segregating family have been used for telocentric mapping. The first method (e.g. Sears, 1962; Law and Wolfe, 1966) uses a backcross family produced by crossing the heterozygous monotelodisomic as the pollen parent on to a euploid plant homozygous for the recessive allele. The second method, described by Driscoll (1966), uses a selfed $F_{2}$ family.

The backcross method has the merit of being more simple to analyse while the selfing method may be more convenient in that suitable $\mathrm{F}_{2}$ grain is easier to produce, wheat being an inbreeder. Also $F_{2}$ grain may already be available from a monosomic analysis using monotelocentrics to identify the critical chromosome, e.g. The and McIntosh (1975). These methods are analogous to the mapping techniques used for two linked loci considered by Mather (1936). However, because of the abnormal meiotic behaviour of telocentric chromosomes in wheat, linkage analysis and the assessment of relative efficiencies of the two methods are more complex. This paper describes the relative precision of the two methods in several genetic situations. 


\section{Comparison of methods}

In the sections below 42 tt denotes ditelocentric plants, $42 \mathrm{t}$, monotelodisomics and 42 , euploid or normal plants. The parameter $p$ denotes the recombination frequency between the centromere and a locus $A 1 / A 2$. In all cases $p$ is assumed to be the same in the male as in the female. The parameter $y$ denotes the transmission frequency of the telocentric through the pollen. In practice this value varies between 0.05 and 0.35 depending apparently both on the telocentric in question and the experimental conditions. Transmission through the female is assumed to be normal, i.e. 0.5 (Sears, 1952). The parameter $n$ denotes the total number of progeny observed.

The phenotypic frequencies in terms of $y$ and $p$ are given in table 1 for backcross and selfed families. The genetic situation referred to as "no dominance" denotes the case where the heterozygote A1/A2 may be distinguished from either homozygote. A further factor that may affect these probabilities is the possibility of reduced viability of 42 tt zygotes. This is considered separately below.

\section{TABle 1}

Probabilities of phenotypes from backcross and selfing methods of telocentric mapping

a. Backcross

e.g. A2 dominant

Phenotype 42

AI $A 2$

$A 1 A 1$

$$
\frac{1}{2} p
$$

b. Selfing
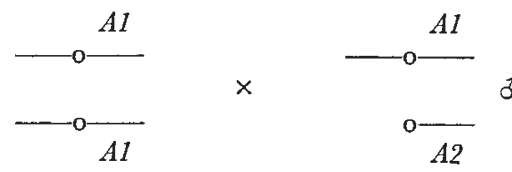

$42 \mathrm{t}$

$\frac{1}{2}(1-p)$
$\frac{1}{2} p$

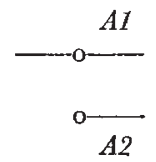

Phenotype 42

(i) $A 1$ dominant

$\begin{array}{lll}A 1 . & a_{1} & \frac{1}{2}(1-y)\left(1-p^{2}\right) \\ A 2 A 2 & a_{4} & \frac{1}{2}(1-y) p^{2}\end{array}$

Total A $\frac{1}{2}(1-y)$

(ii) $A 2$ dominant

A2. $a_{1} \frac{1}{2}(1-y)\left(1-(1-p)^{2}\right)$

$A 1 \quad A 1 \quad a_{4} \quad \frac{1}{2}(1-y)(1-p)^{2}$

Total $A \frac{1}{2}(1-y)$

(iii) No dominance

$A I A I \quad a_{1} \frac{1}{2}(1-y)(1-p)^{2}$

$A 1 A 2 \quad a_{4} \quad(1-y) p(1-p)$

$A 2 \mathrm{A2} \quad a_{7} \quad \frac{1}{2}(1-y) p^{2}$

Total A $\frac{1}{2}(1-y)$

$a_{1}$ to $a_{9}$ and $\mathrm{A}, \mathrm{B}, \mathrm{C}$ denote observed numbers in the various classes.
$42 \mathrm{tt}$

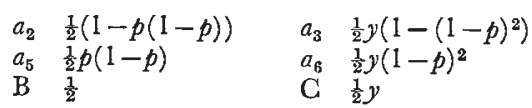

$$
\begin{array}{llll}
a_{2} & \frac{1}{2}(1-p(1-p)) & a_{3} & \frac{1}{2} y\left(1-p^{2}\right) \\
a_{5} & \frac{1}{2} p(1-p) & a_{6} & \frac{1}{2} y p^{2} \\
\mathrm{~B} & \frac{1}{2} & \mathrm{C} & \frac{1}{2} y
\end{array}
$$

$$
\begin{array}{llll}
a_{2} & \frac{1}{2} p(1-p) & a_{3} & \frac{1}{2} y p^{2} \\
a_{5} & \frac{1}{2}\left(1-2 p+2 p^{2}\right) & a_{6} & y p(1-p) \\
a_{8} & \frac{1}{2} p(1-p) & a_{9} & \frac{1}{2} y(1-p)^{2} \\
\mathrm{~B} & \frac{1}{2} & \mathrm{C} & \frac{1}{2} y
\end{array}
$$




\section{(i) Detection of the critical arm}

The essential first step in telocentric mapping is to identify the critical arm, assuming prior identification of the critical chromosome by monosomic analysis or other location techniques available in wheat (Law and Worland, 1972). Often identification is obvious from observation of the $42 \mathrm{tt}$ stocks. However, if the allele carried on the telocentric is recessive and a null allele, e.g. rht 2 (McVittie, Gale, Marshall and Westcott, 1978) then only observation of the result of a recombination between the centromere and the locus will identify the critical arm.

In a backcross family the probability that one plant among $n$ progeny is such a recombinant is $1-(1-p)^{n}$. In the selfing method when $A 1$ is dominant this probability is reduced to $1-\left(1-\frac{1}{2} p(1+2 y(1-p))\right)^{n}$ and consequently more plants must be examined. On the other hand, in the case of no dominance the probability is $1-\left(1-\frac{1}{2} p(4-3 p)\right)^{n}$, which is greater than that for a backcross.

\section{(ii) Linkage estimation}

The parameters $y$ and $p$ are estimated using the technique of maximumlikelihood scoring (see Appendix 1 of Bailey (1961)).

Backcross method. The recombination fraction $p$ is estimated by the fraction of total progeny exhibiting recombinant phenotypes. The expected variance of this estimate is $V_{b}=p(1-p) / n$.

Selfing method: A1 dominant. The estimates of $y$ and $p$ with their variances are derived in the Appendix. The ratio $R$ of the expected variances of $p$ in the backcross and selfing methods is a measure of the relative efficiency of the two methods. In this case it simplifies to

$$
R=\frac{V_{b}}{V_{s}}=\frac{2 y(1-2 p)}{(1+p)(2-p)}+\frac{8 p^{3}-4 p^{2}+p+1}{2\left(1+p^{3}\right)}
$$

The minimum and maximum values of $R$ are 0.5 and 1 respectively. Thus, when $A 1$ is dominant, precision of estimation is always less with the selfing than with the backcross method. In the worst case, to obtain as good a standard error for $p$ by selfing, $\sqrt{2}(=1 \cdot 414)$ times as many plants need to be examined compared with backcrossing.

Selfing method: A2 dominant. In this case

$$
R=\frac{2(1-y)(1-2 p)}{(1+p)(2-p)}+\frac{8 p^{3}-4 p^{2}+p+1}{2\left(1+p^{3}\right)}
$$

The maximum value of $R$ is now 1.5 and $R$ is greater than 1 if $y$ and $p$ are reasonably small. For example, if $y=0 \cdot 1, R>1$ provided $p<0 \cdot 228$. The minimum value of $R$ is $\frac{2}{3}$, achieved when $p=\frac{1}{2}$. Thus, when $A 2$ is dominant, the selfing method may be more efficient for linkage estimation than the backcross method and is never grossly less efficient.

Selfing method: no dominance. In this case $R=2\left(1-3 p+3 p^{2}\right) /\left(1-2 p+2 p^{2}\right)$ and no longer involves $y$. This result was obtained by Mather (1936) for the case of two loci each with two alleles exhibiting incomplete dominance. This, of course, is because the segregation of the telocentric and complete chromosomes is statistically analogous to that of a gene not exhibiting 
dominance. It is shown in the Appendix that $R$ is here never less than 1 . Thus, when there is no dominance, linkage values are always estimated more precisely by the selfing method than the backcross method.

The effect of the three $A 1 / A 2$ dominance relationships on the relative precision of linkage estimation obtained by the two methods is illustrated in fig. 1 for a typical value of $y$.

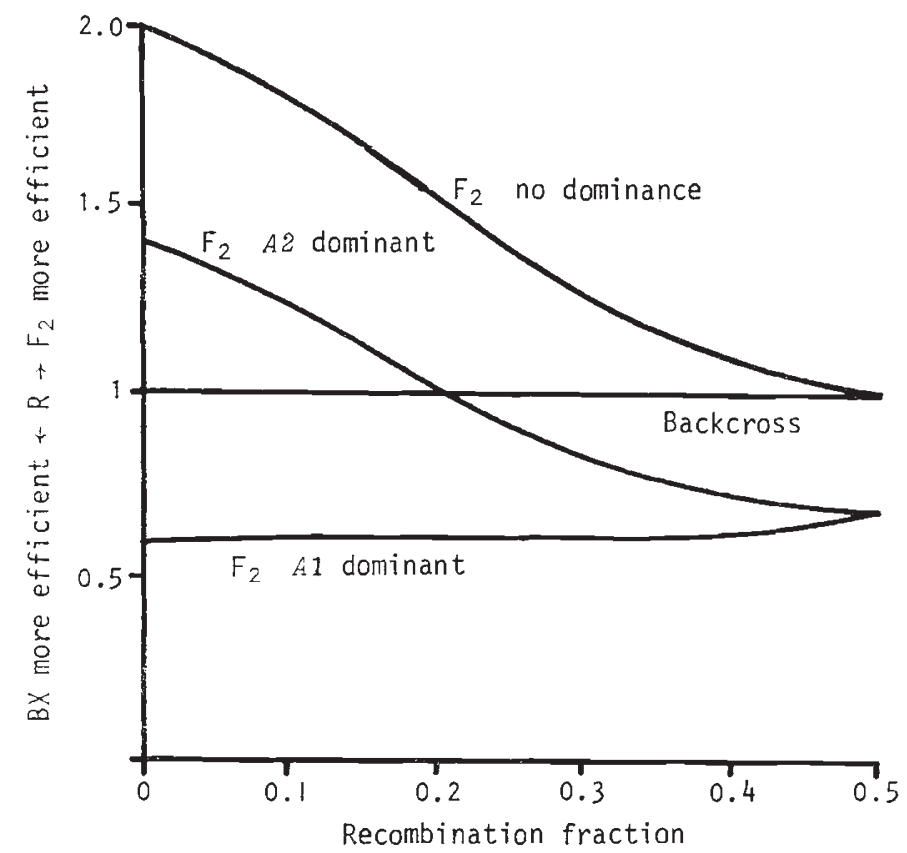

Fig. 1.-Relative efficiencies of the selfing and backcross methods of telocentric mapping when male transmission is $0 \cdot 1$. The no dominance situation is unaffected by this condition.

\section{(iii) Reduced zygotic viability}

In analysing $\mathrm{F}_{2}$ data, the effect of differential male transmission is to reduce the expected number of $42 \mathrm{tt}$ plants from $\frac{1}{4} n$ to $\frac{1}{2} y n$. For certain chromosomes this number may be reduced still further through reduced viability of the 42 tt zygotes.

A simple statistical test for this phenomenon is derived in the Appendix. Reduced zygotic viability will be detected at the 5 per cent level of significance if $(B-A-C-1) / \sqrt{n}$ is greater than $1 \cdot 64$, the 95 per cent point of the standard normal distribution.

If $y$ is small, correspondingly few 42 tt plants will be expected because of the effects of reduced transmission alone. In this case it will be more difficult to detect any extra effect of reduced zygotic viability (table 2 of the Appendix).

In analysing $\mathrm{F}_{2}$ data when $y$ is small and $B>\frac{1}{2} n$ the standard error of $p$ should be calculated using equations (4) or (6) of the Appendix (depending on the dominance relationship between $A 1$ and $A 2$ ) as well as (2) or (3). 
If these standard errors are appreciably different they should both be quoted as the viability of ditelocentric zygotes may be reduced but this is not being detected with the size of sample available.

In all three dominance situations one consequence of not considering reduced zygotic viability when it is operating is underestimation of $y$. When $A 1$ is dominant, reduced zygotic viability reduces the precision of estimation of $p$, the reduction in precision increasing with the reduction in viability. The minimum value of $R$ in this case becomes 0.416 . When $A 2$ is dominant reduced zygotic viability always increases the precision of linkage estimation when the recombination fraction is below $0 \cdot 245$. Above this value, decreases in viability decrease the precision most when $y$ and $p$ approach 0.5 . The minimum value of $R$ is then 0.444 (when $y=0.5$ and $p=0.5$ ). In the no dominance case reduced zygotic viability always reduces the precision of linkage estimation, the minimum value of $R$ being 0.667 . However, if $p<0 \cdot 3, R>1$ irrespective of any reduced zygotic viability effect and $R>1$ for $p>0.3$ providing this viability effect is not too great.

\section{Planning of experiments}

The above considerations may be of some help to the experimenter using the selfing method in deciding the number of $F_{2}$ plants to examine. Unless the recombination fraction is extremely small the problem of detecting the critical arm is likely to be secondary. For example, if the probability of a plant being recognised as a recombinant is greater than 0.03 the probability of observing at least one recombinant among $100 \mathrm{~F}_{2}$ progeny exceeds 0.95 .

The decision on the size of $F_{2}$ progeny required to obtain a given precision for estimation of linkage depends on the experimenter's prior knowledge of $y$ and $p$ and whether the viability of $42 \mathrm{tt}$ plants is likely to be reduced. If a rough idea of their values is known an approximate value for the standard error of $p$ can be calculated. A guide to the number of plants required to produce a standard error $s$ for the estimate of $p$ is then $1 / s^{2} I_{p p}$, using equations (2), (3), (4) or (6) of the Appendix. If no prior knowledge is available, $V_{s} \leqq V_{b} / M=\left(p-p^{2}\right) / M n<1 / 4 M n$, where $M$ is the minimum value of $R$, quoted above, for the particular dominance relationship between $A 1$ and $A 2$ taking into account any reduction in zygotic viability. Thus, in order to guarantee a standard error of $s, 1 / 4 M s^{2}$ plants need to be examined.

As an example, assume an experimenter is dealing with a character where $A 1$ is completely dominant and differential viability is discounted (and so $M=0.5$ ). If the experimenter has no idea what the probable values of $p$ or $y$ might be, then, in order to guarantee a standard error of 0.05 or less for the estimate of $p$, he or she will need to examine $1 /\left(4 \times 0.5 \times 0.05^{2}\right)=200$ plants.

If $A 2$ was completely dominant this number would be reduced to 150 while in the case of no dominance only 100 plants would be necessary.

It is emphasised that these numbers give a guaranteed upper bound for the standard error of $p$. In practice, of course, the estimated standard error will almost certainly be less than the guaranteed value, depending on the values of $p$ and $y$ actually obtained.

All the calculations on $\mathrm{F}_{2}$ data described in this paper can be performed by a computer program, written in standard Fortran, which is available from the senior author. 


\section{REFERENCES}

BaIley, N. T. J. 1961. Introduction to the Mathematical Theory of Genetic Linkage. Oxford University Press.

DRISCOLL, C. J. 1966. Gene-centromere distance in wheat by aneuploid $\mathbf{F}_{2}$ observations. Genetics, 54, 131-135.

LAW, C. N., AND WOLFE, M. s. 1966. Location of genetic factors for mildew resistance and ear emergence time on chromosome 7B of wheat. Can. F. Genet. Cytol., 8, 462-470.

LAW, C. N., AND WORLAND, A. J. 1972. Aneuploidy in wheat and its uses in genetic analysis. Plant Breeding Institute Annual Report, 26-65.

mather, к. 1936. Types of linkage data and their value. Ann. Eugenics, 7, 251-264.

MGVITTIE, J. A., GALE, M. D., MARSHALL, G. A., AND WESTCOTT, B. 1978. The intra-chromosomal mapping of the Norin 10 and Tom Thumb dwarfing genes. Heredity, 40, 67-70. SEARs, E. R. 1952. The behaviour of isochromosomes and telocentrics in wheat. Chromosoma, $4,551 \sim 562$.

SEARS, E. R. 1962. The use of telocentric chromosomes in linkage mapping. Genetics, 47, 983. THE, T. T., AND ManTosh, R. A. 1975. Cytogenetical studies in wheat. VIII. Telocentric mapping and linkage studies involving Sr22 and other genes in chromosome 7AL. Aust. 7. of Biol. Sci., 28, 531-538.

\section{APPENDix}

\section{(i) Linkage estimation}

When $A 1$ is dominant the scores for $y$ and $p$ are

$$
\begin{aligned}
S_{y}=-A /(1-y)+C / y & \\
S_{p}=-2 a_{1} p /\left(1-p^{2}\right)- & a_{2}(1-2 p) /\left(1-p+p^{2}\right)+2 a_{3}(1-p) /(p(2-p)) \\
& +2 a_{4} / p+a_{5}(1-2 p) /(p(1-p))-2 a_{6} /(1-p)
\end{aligned}
$$

as used by The and McIntosh (1975). The information matrix is

where

$$
n\left[\begin{array}{ll}
I_{y y} & I_{y p} \\
I_{y p} & I_{p p}
\end{array}\right],
$$

and

$$
I_{y y}=1 / 2 y(1-y), \quad I_{y p}=0
$$

$$
\begin{aligned}
I_{p p}=2(1-y)\left(p^{2} /\left(1-p^{2}\right)\right. & +1)+2 y\left((1-p)^{2} /\left(2 p-p^{2}\right)+1\right) \\
& +\frac{1}{2}(2 p-1)^{2}\left(1 /\left(1-p+p^{2}\right)+1 /(p(1-p))\right) .
\end{aligned}
$$

The equation $S_{y}=0$ gives $y=C /(A+C)$ with variance $1 / n I_{y y}$. The equation $S_{p}=0$ is solved by the usual iterative method to give the estimate of $p$ with variance $V_{s}=1 / n I_{p p}$.

When $A 2$ is dominant $S_{p}$ and $I_{p p}$ are obtained by substituting $1-p$ for $p$ in (1) and (2) as can be seen by comparing tables $1 \mathrm{~b}$ (i) and $1 \mathrm{~b}$ (ii).

When there is no dominance,

and

$$
\begin{aligned}
S_{p}=-2\left(a_{1}+a_{9}\right) /(1-p) & +\left(a_{2}+a_{4}+a_{6}+a_{8}\right)(1-2 p) /\left(p-p^{2}\right) \\
& +2\left(a_{3}+a_{7}\right) / p+a_{5}(4 p-2) /\left(1-2 p+2 p^{2}\right)
\end{aligned}
$$

Thus

$$
I_{p p}=2 /\left(p-p^{2}\right)-2 /\left(1-2 p+2 p^{2}\right)
$$

$$
R=V_{b} / V_{s}=2-2\left(p-p^{2}\right) /\left(1-2 p+2 p^{2}\right) \geqq 1
$$


if $2\left(p-p^{2}\right) /\left(1-2\left(p-p^{2}\right)\right) \leqq 1$, i.e. if $p-p^{2} \leqq \frac{1}{4}$ or $4 p^{2}-4 p+1 \geqq 0$ which is always true since the left-hand side is $(2 p-1)^{2}$. Hence $R \geqq 1$ in this case.

\section{(ii) Reduced zygotic viability}

The relative proportions of the chromosomal classes $42: 42 \mathrm{t}: 42 \mathrm{tt}$ can be represented in a realistic model by $w(1-y): w: 1-2 w+w y$ where $w$ is the proportion of $42 \mathrm{t}$ plants. Maximum likelihood estimates of $w$ and $y$ are $B / n$ and $(B-A) / B$. If there is reduced zygotic viability, the observed number of $42 \mathrm{tt}$ plants is less than the number expected, so that $C<\frac{1}{2} n(B-A) / B$, i.e. $B>A+C$. The difference between the two estimates of $y$ is $(B-A) / B-C /(A+C)=A(B-A-C) /(B(A+C))>0$ so $y$ is underestimated by $C /(A+C)$ when reduced zygotic viability is present. $B>A+C$ implies the estimate of $w, B / n>\frac{1}{2}$. Under the null hypothesis $w=\frac{1}{2}$ (against the alternative $w>\frac{1}{2}$ ) the number of monotelodisomics has a binomial distribution with parameters $\left(\frac{1}{2}, n\right)$ which has expectation $\frac{1}{2} n$ and variance $\frac{1}{4} n$. Using the normal approximation to the binomial and applying a continuity correction, $\left(\left|B-\frac{1}{2} n\right|-\frac{1}{2}\right) / \frac{1}{2} \sqrt{n}=(B-A-C-1) / \sqrt{n}$ is an observed value of a random variable which has approximately a standard normal distribution if the null hypothesis is true. Since $B / n$ estimates $w$, reduced zygotic viability will be detected at the 5 per cent significance level if $((2 w-1) n-1) / \sqrt{n} \geqq 1 \cdot 64$. Solving this quadratic inequality for $n$ gives the results for various values of $w$ shown in table 2 .

TABLE 2

For various proportions of monotelodisomics, w the table provides the minimum sample size, $\mathrm{n}$, needed to detect reduced zygotic viability at the 5 per cent level of significance and the minimum possible male transmission frequency, $\mathrm{y}$

\begin{tabular}{ccr}
$w$ & $y$ & \multicolumn{1}{c}{$n$} \\
0.5000 & $0 \cdot 0$ & - \\
0.5263 & 0.1 & 1014 \\
0.5556 & 0.2 & 237 \\
0.5882 & 0.3 & 98 \\
0.6250 & 0.4 & 51 \\
0.6667 & 0.5 & 31
\end{tabular}

Since the proportion of $42 \mathrm{tt}$ plants, $1-2 w+w y \geqq 0, y \geqq 2-1 / w$. This gives the minimum value of $y$ for each $w$.

When $A 1$ is dominant,

$$
I_{p p}=\frac{1}{2-p}\left[\frac{4}{p}-\frac{4 w(1-y)(1-2 p)}{p(1-p)(1+p)}-\frac{w\left(2+p-4 p^{2}\right)}{p(1-p)\left(1-p+p^{2}\right)}\right] .
$$

Denoting the value when $w=\frac{1}{2}$ by $I_{p p}^{\prime}$,

$$
I_{p p}^{\prime}-I_{p p}=\frac{4\left(w-\frac{1}{2}\right)(1-y)(1-2 p)}{p(1-p)(1+p)(2-p)}+\frac{\left(w-\frac{1}{2}\right)\left(2+p-4 p^{2}\right)}{p(1-p)\left(1-p+p^{2}\right)(2-p)},
$$

which is positive for $w>\frac{1}{2}$ and $0 \leqq p \leqq \frac{1}{2}$. As before, when $A 2$ is dominant, $p$ in (4) and (5) is replaced by $1-p$, or equivalently the behaviour of these 40/1-E 
expressions may be examined for $0.5 \leqq p \leqq 1 \cdot 0$, where the recombination fraction is now $1-p$.

When $p>0.5$, the first term on the right-hand side of $(5)$ is negative. The second term is negative for $p>0.843$. For $0.5 \leqq p<0.843$ the sign of $I_{p p}^{\prime}-I_{p p}$ depends on the relative absolute magnitude of the two terms. It will be negative when $4(1-y)(2 p-1) /(1+p)>\left(2+p-4 p^{2}\right) /\left(1-p+p^{2}\right)$. If this inequality holds for $y=\frac{1}{2}$ it holds a fortiori for $y<\frac{1}{2}$. Putting $y=\frac{1}{2}$ gives $3 p^{3}-3 p^{2}+3 p-4>0$ and solving the cubic gives $p>0 \cdot 755$, i.e. the recombination fraction $<0 \cdot 245$.

When there is no dominance,

$$
I_{p p}=2 /\left(p-p^{2}\right)-4 w /\left(1-2 p+2 p^{2}\right) .
$$

Thus when $w>\frac{1}{2}, I_{p p}$ is less than its value given by (3) and the estimated variance of $p$ is increased when $w>\frac{1}{2}$. 